\title{
OSCILLATION OF IMPULSIVE DELAY DIFFERENTIAL EQUATIONS AND APPLICATIONS TO POPULATION DYNAMICS
}

\author{
JURANG YAN ${ }^{1}$, AIMIN ZHAO ${ }^{1}$ and LINPING PENG ${ }^{2}$
}

(Received 8 January, 2004; revised 14 April, 2004)

\begin{abstract}
The main result of this paper is that the oscillation and nonoscillation properties of a nonlinear impulsive delay differential equation are equivalent respectively to the oscillation and nonoscillation of a corresponding nonlinear delay differential equation without impulse effects. An explicit necessary and sufficient condition for the oscillation of a nonlinear impulsive delay differential equation is obtained.
\end{abstract}

\section{Introduction}

The theory of impulsive differential equations is emerging as an important area of investigation, since it is far richer than the corresponding theory of differential equations without impulse effects. Moreover, such equations may be used to model several real-world phenomena in areas such as physics, biology and engineering. In the last twenty years, the theory of impulsive ordinary differential equations and delay differential equations has been studied by many authors. We refer, in particular, to the monographs $[7,8,10-12]$. In recent years, the theory of impulsive delay differential equations has attracted the attention of many mathematicians and numerous papers have been published on this class of equations (see $[2-6,9,13,15]$ and references therein).

Let $\mathscr{N}=\{1,2,3, \ldots\}$ and $\left\{t_{k}\right\}_{k=1}^{\infty}$ be a monotone increasing unbounded sequence of positive numbers. Consider the nonlinear delay differential equation

$$
y^{\prime}(t)+\sum_{i=1}^{m} f_{i}\left(t, y\left(g_{i}(t)\right)\right)=0, \quad t \neq t_{k}, \text { a.e. (almost everywhere), } t \geq 0,
$$

\footnotetext{
'School of Mathematical Sciences, Shanxi University, Taiyuan, Shanxi 030006, People's Republic of China; e-mail: jryan@sxu.edu.cn.

${ }^{2}$ School of Science, Beijing University of Aeronautics and Astronautics, Beijing 100083, People's

Republic of China.

(C) Australian Mathematical Society 2005, Serial-fee code 1446-1811/05
} 
with the impulsive conditions

$$
y\left(t_{k}^{+}\right)-y\left(t_{k}\right)=b_{k} y\left(t_{k}\right), \quad k \in \mathscr{N},
$$

under the following hypotheses:

( $\left.\mathrm{A}_{1}\right) f_{i} \in([0, \infty) \times R, R), i=1,2, \ldots, m$, are Lebesgue measurable and essentially bounded in each finite interval $[0, b]$ in $t$ for each fixed $u$ and are continuous in $u$ for each fixed $t$. Moreover, $u f_{i}(t, u)>0$ for all $u \neq 0$ and $t>0$, and $f_{i}(t, u)$ are nondecreasing in $u, i=1,2, \ldots, m$.

$\left(\mathrm{A}_{2}\right) \quad g_{i}(t) \in([0, \infty),[0, \infty))$ are Lebesgue measurable and $g_{i}(t) \leq t, \lim _{t \rightarrow \infty} g_{i}(t)=\infty$, $i=1,2, \ldots, m$.

$\left(\mathrm{A}_{3}\right) \quad b_{k} \in(-1, \infty)$ are constants, $k \in \mathscr{N}$.

For every $\sigma \geq 0$, we define throughout this paper

$$
r_{\sigma}=\min _{1 \leq i \leq m} \inf _{t \geq \sigma}\left\{g_{i}(t)\right\}
$$

and let $\Phi_{\sigma}$ denote the set of functions $\phi:\left[r_{\sigma}, \sigma\right] \rightarrow R$ which are real valued and Lebesgue measurable.

DEFINITION 1. For given $\sigma \geq 0$ and $\phi \in \Phi_{\sigma}$, a function $y(t) \in\left(\left[r_{\sigma}, \infty\right), R\right)$ is said to be a solution of (1.1) with (1.2) on $\left[r_{\sigma}, \infty\right)$ satisfying the initial value condition

$$
y(t)=\phi(t), \quad t \in\left[r_{\sigma}, \sigma\right],
$$

if the following conditions are satisfied:

(i) $y(t)$ is defined on $\left[r_{\sigma}, \infty\right)$ and satisfies (1.4) on $\left[r_{\sigma}, \sigma\right]$;

(ii) $y(t)$ is absolutely continuous on each interval $\left(\sigma, t_{K}\right)$ and $\left(t_{K}, t_{K+1}\right),\left(t_{K+1}\right.$, $\left.t_{K+2}\right), \ldots$, where $t_{K}=\min \left\{t_{k}: t_{k}>\sigma, k \in \mathscr{N}\right\}$;

(iii) for each $t_{k} \geq t_{K}, y\left(t_{k}^{+}\right), y\left(t_{k}^{-}\right)$exist and $y\left(t_{k}^{-}\right)=y\left(t_{k}\right)$;

(iv) $y(t)$ satisfies $(1.1)$ a.e. in $(\sigma, \infty) \backslash\left\{t_{k}\right\}$ and satisfies the impulsive condition (1.2).

DEFINITION 2. A solution of (1.1) with (1.2) is said to be oscillatory if it is neither eventually positive nor eventually negative. Otherwise, it is called nonoscillatory.

Under appropriate conditions, global existence and uniqueness theorems for solutions of the initial value problem (1.1) with (1.2) on $[\sigma, \infty)$ have been established in [13].

The purpose of this paper is to study the oscillatory and nonoscillatory behaviour of solutions of the first-order nonlinear impulsive delay differential equation (1.1) with (1.2). An explicit necessary and sufficient condition for the oscillation of all solutions of (1.1) with (1.2) is obtained. Our results will be nonlinear in nature as we stress the role played by the nonlinear terms in the oscillation of (1.1) with (1.2) and the known oscillation criteria cannot be applied to the form of (1.1) with or without (1.2). 


\section{Main results}

Consider the following auxiliary equation for $t \geq \sigma \geq 0$ :

$$
x^{\prime}(t)+\sum_{i=1}^{m} \prod_{\sigma<t_{k}<t}\left(1+b_{k}\right)^{-1} f_{i}\left(t, \prod_{\sigma<t_{k}<g_{i}(t)}\left(1+b_{k}\right) x\left(g_{i}(t)\right)\right)=0 \text { a.e. }
$$

Here and in what follows we assume that a product equals unity if the number of factors is equal to zero.

By a solution $x(t)$ of $(*)$ on $\left[r_{\sigma}, \infty\right), \sigma \geq 0$, we mean an absolutely continuous function $x(t)$ on $[\sigma, \infty)$ which satisfies $(*)$ on $[\sigma, \infty)$ a.e. and has the initial condition $x(t)=\phi(t)$ on $\left[r_{\sigma}, \sigma\right]$ where $r_{\sigma}$ is defined by (1.3) and $\phi \in \Phi_{\sigma}$.

Under appropriate hypotheses the theory of existence and uniqueness of the initial value problem (*) with (1.4) can be obtained by the method of steps (see [10, page 5]).

A solution of $(*)$ is said to be oscillatory if it has arbitrarily large zeros. Otherwise, the solution is called nonoscillatory.

In this section, we first establish the following lemma which is a new result.

LEMMA 2.1. Assume that $\left(\mathrm{A}_{1}\right)-\left(\mathrm{A}_{3}\right)$ hold. Let $\sigma \geq 0$ and $\phi \in \Phi_{\sigma}$. Then (1.1)-(1.2) has a solution $y(t)$ on $\left[r_{\sigma}, \infty\right)$ if and only if $(*)$ has a solution $x(t)$ on $\left[r_{\sigma}, \infty\right)$.

PROOF. Suppose that $y(t)$ is a solution of (1.1) with $(1.2)$ on $\left[r_{\sigma}, \infty\right)$. Set

$$
x(t)=\prod_{\sigma<t_{k}<t}\left(1+b_{k}\right)^{-1} y(t), \quad t \geq \sigma \quad \text { and } \quad x(t)=y(t), \quad r_{\sigma} \leq t \leq \sigma .
$$

Thus $x(t)=y(t)=\phi(t), t \in\left[r_{\sigma}, \sigma\right]$. By taking into account the fact that $y(t)$ is absolutely continuous on each interval $\left(\sigma, t_{K}\right)$ and $\left(t_{K}, t_{K+1}\right),\left(t_{K+1}, t_{K+2}\right), \ldots$ and (2.1) we have that for each $t_{k} \geq t_{K}$,

$$
x\left(t_{k}^{+}\right)=\prod_{\sigma<t_{j} \leq t_{k}}\left(1+b_{j}\right)^{-1} y\left(t_{k}^{+}\right)=\prod_{\sigma<t_{j}<t_{k}}\left(1+b_{j}\right)^{-1} y\left(t_{k}\right)=x\left(t_{k}\right)
$$

and

$$
x\left(t_{k}^{-}\right)=\prod_{\sigma<t_{j} \leq t_{k}}\left(1+b_{j}\right)^{-1} y\left(t_{k}^{-}\right)=\prod_{\sigma<t_{j}<t_{k}}\left(1+b_{j}\right)^{-1} y\left(t_{k}\right)=x\left(t_{k}\right) .
$$

Hence $x(t)$ is continuous on $[\sigma, \infty)$. Since $y(t)$ is absolutely continuous on each interval $\left(\sigma, t_{K}\right)$ and $\left(t_{K}, t_{K+1}\right), \ldots$, it is easy to prove that $x(t)$ is also absolutely continuous on $[\sigma, \infty)$. Moreover, from (1.1) and (2.1) we have that $(*)$ holds for $t \geq \sigma$. Conversely, suppose that $x(t)$ is a solution of $(*)$ on $\left[r_{\sigma}, \infty\right)$. Let

$$
y(t)=\prod_{\sigma<t_{k}<t}\left(1+b_{k}\right) x(t), \quad t>\sigma, \quad y(t)=x(t), \quad r_{\sigma} \leq t \leq \sigma .
$$


Then from (*) and (2.2) we have that for $t>\sigma$

that is,

$$
\prod_{\sigma<t_{k}<t}\left(1+b_{k}\right)^{-1} y^{\prime}(t)+\sum_{i=1}^{m} \prod_{\sigma<t_{k}<t}\left(1+b_{k}\right)^{-1} f\left(t, y\left(g_{i}(t)\right)\right)=0 \quad \text { a.e. }
$$

$$
y^{\prime}(t)+\sum_{i=1}^{m} f_{i}\left(t, y\left(g_{i}(t)\right)\right)=0 \quad \text { a.e. }
$$

which implies that $y(t)$ satisfies (1.1). On the other hand, from (2.2), for each $t_{k} \geq t_{K}$,

$$
y\left(t_{k}^{+}\right)=\lim _{t \rightarrow t_{k}^{+}} \prod_{\sigma<t_{j}<t}\left(1+b_{j}\right) x(t)=\prod_{\sigma<t_{j} \leq t_{k}}\left(1+b_{j}\right) x\left(t_{k}\right)
$$

and $y\left(t_{k}\right)=\prod_{\sigma<t_{j}<t_{k}}\left(1+b_{j}\right) x\left(t_{k}\right)$. So, for each $t_{k} \geq t_{K}, y\left(t_{k}^{+}\right)=\left(1+b_{k}\right) y\left(t_{k}\right)$. Thus $y(t)$ satisfies the impulsive condition (1.2). The proof of Lemma 2.1 is complete.

From Lemma 2.1, we can obtain the following comparison theorem.

THEOREM 2.2. Assume that $\left(\mathrm{A}_{1}\right)-\left(\mathrm{A}_{3}\right)$ hold. Then all solutions of $(1.1)$ with (1.2) are oscillatory if and only if all solutions of $(*)$ are oscillatory.

In the following theorem, we give a sufficient condition for (1.1) with (1.2) to have an eventually positive solution.

THEOREM 2.3. Assume that $\left(\mathrm{A}_{1}\right)-\left(\mathrm{A}_{3}\right)$ hold and for any constant $\beta>0$ such that

$$
\int_{0}^{\infty} \sum_{i=1}^{m} \prod_{0<i_{k}<t}\left(1+b_{k}\right)^{-1} f_{i}\left(t, \beta \prod_{0<l_{k}<8_{i}(t)}\left(1+b_{k}\right)\right) d t<\infty,
$$

then (1.1) with (1.2) has an eventually positive solution.

Proof. From Lemma 2.1 , we need to prove that $(*)$ has an eventually positive solution. Let $\sigma \geq 0$, then by (2.3) we can choose $T>0$ such that

$$
0<\int_{T}^{\infty} \sum_{i=1}^{m} \prod_{\sigma<i_{k}<t}\left(1+b_{k}\right)^{-1} f_{i}\left(t, \bar{\beta} \prod_{\sigma<t_{k}<\beta_{i}(t)}\left(1+b_{k}\right)\right) d t<\bar{\beta},
$$

where $\bar{\beta}=\beta \prod_{0<t_{t} \leq \sigma}\left(1+b_{k}\right)$. Let $T_{0}=\min _{1 \leq i \leq m} \inf _{t \geq r}\left\{g_{i}(t)\right\}$ and $X$ denote the locally convex space of all continuous functions $x \in\left(\left[T_{0}, \infty\right), R\right)$ with the topology of uniform convergence on a compact subinterval of $\left[T_{0}, \infty\right)$. Let $M=\{x \in X$ : 
$\bar{\beta} \leq x(t) \leq 2 \bar{\beta}, t \geq T_{0}$ \}. Observe that $M$ is a closed convex subset of $X$. Define the operator $F$ by

$$
(F x)(t)=\left\{\begin{aligned}
2 \bar{\beta}- & \int_{T}^{t} \sum_{i=1}^{m} \prod_{\sigma<t_{k}<s} \frac{f_{i}\left(s, \prod_{\sigma<t_{k}<g_{i}(s)}\left(1+b_{k}\right) x\left(g_{i}(s)\right)\right)}{\left(1+b_{k}\right)} d s, \\
t & \geq T, \\
2 \bar{\beta}, \quad T_{0} & \leq t<T .
\end{aligned}\right.
$$

We now prove that

(i) for any $x \in M,(F x)(t)$ is continuous on $\left[T_{0}, \infty\right)$;

(ii) $F$ maps $M$ continuously into a compact subset of $M$.

For any $t_{0} \in(T, \infty)$ or $t_{0} \in\left[T_{0}, T\right)$, we have $\lim _{t \rightarrow t_{0}}(F x)(t)=(F x)\left(t_{0}\right)$ and $\lim _{t \rightarrow T^{+}}(F x)(t)=\lim _{t \rightarrow T^{-}}(F x)(t)=2 \bar{\beta}$, which imply that $F$ is a continuous function on $\left[T_{0}, \infty\right)$. This proves (i).

If for any $x \in M$, in view of (2.4) and (2.5), we obtain $\bar{\beta} \leq(F x)(t) \leq 2 \bar{\beta}, t \geq T_{0}$. Thus $(F x)(t) \in M$. Also, $(F x)(t), x \in M$, are uniformly bounded and since for any $x \in M$ and $t_{2}>t_{1} \geq T_{0}$

$$
\begin{aligned}
\left|(F x)\left(t_{2}\right)-(F x)\left(t_{1}\right)\right| & \leq \int_{t_{1}}^{t_{2}} \sum_{i=1}^{m} \prod_{\sigma<t_{k}<s} \frac{f_{i}\left(s, \prod_{\sigma<t_{k}<8_{i}(s)}\left(1+b_{k}\right) x\left(g_{i}(s)\right)\right)}{\left(1+b_{k}\right)} d s \\
& \leq \bar{\beta}\left(t_{2}-t_{1}\right),
\end{aligned}
$$

it follows from (2.5) that $\{F x\}$ are equicontinuous on any compact subintervals of $\left[T_{0}, \infty\right)$. Therefore $F$ maps $M$ continuously into a compact subset of $M$. This proves (ii).

Consequently, by the Schauder-Tychonov fixed point theorem, $F$ has a fixed point $x$ in $M$. Obviously, this fixed point $x=x(t)$ is a positive solution of $(*)$ on $\left[T_{0}, \infty\right)$. By Lemma 2.1, $y(t)=\prod_{T_{0<t_{k}<t}}\left(1+b_{k}\right) x(t)$ is a positive solution of (1.1) with (1.2) on $\left[T_{0}, \infty\right)$. The proof of Theorem 2.3 is complete.

REMARK. Similarly, we can prove that if for any constant $\beta>0$

$$
\int_{0}^{\infty} \sum_{i=1}^{m} \prod_{0<t_{k}<1}\left(1+b_{k}\right)^{-1} f_{i}\left(t,-\beta \prod_{0<t_{k}<g_{i}(t)}\left(1+b_{k}\right)\right) d s>-\infty,
$$

then (1.1) with (1.2) has an eventually negative solution.

DEFINITION 3. A solution $y(t)$ of $(1.1)$ with $(1.2)$ on $\left[T_{y}, \infty\right)$ is said to be a proper solution if for all sufficiently large $t, \sup _{t \geq T}|y(t)|>0$ for any $T \geq T_{y}>\sigma$, that is, for any sufficiently large $T \geq T_{y}>\sigma,|y(t)| \not \equiv 0$ on $[T, \infty)$. 
LEMMA 2.4. Assume that $\left(\mathrm{A}_{1}\right)-\left(\mathrm{A}_{3}\right)$ hold. Then (1.1) with (1.2) has a proper solution if and only if $(*)$ also has a proper solution.

PROOF. Let $y(t)$ be a proper solution of (1.1) with (1.2) on $\left[T_{y}, \infty\right)$. From Lemma 2.1, $x(t)=\prod_{T_{y}<t_{k}<t}\left(1+b_{k}\right)^{-1} y(t)$ is a solution of $(*)$ on $\left[T_{y}, \infty\right)$. Suppose that $(*)$ does not have a proper solution. From Definition 3 there exists a sufficiently large $T_{x} \geq T_{y}$ such that $x(t) \equiv 0$ on $\left[T_{x}, \infty\right)$. Thus $y(t)=\prod_{T_{x}<t_{k}<t}\left(1+b_{k}\right) x(t) \equiv 0$ on $\left[T_{x}, \infty\right)$, which contradicts the fact that $y(t)$ is a proper solution. Similarly, we can prove that if $(*)$ has a proper solution, then (1.1) with (1.2) also has a proper solution. The proof of Lemma 2.4 is now complete.

REMARK. In what follows, we will only consider proper solutions of (1.1) with (1.2) or $(*)$.

We introduce the following locally sublinear condition near $u=0$.

$\left(\mathrm{A}_{4}\right)$ There exist functions $p_{i}(t) \in([0, \infty),[0, \infty)), i=1,2, \ldots, m$, which are Lebesgue measurable and essentially bounded in each finite interval, and constants $\delta>0,0<\alpha<1$ such that for all sufficiently large $t$ and $0<|u|<\delta$,

$$
\inf _{\delta>|u|>0} \frac{f_{i}(t, u)}{u|u|^{\alpha-1}} \geq p_{i}(t)
$$

THEOREM 2.5. Assume that $\left(\mathrm{A}_{1}\right)-\left(\mathrm{A}_{4}\right)$ hold and

$$
\int_{0}^{\infty} \sum_{i=1}^{m} \prod_{0<t_{k}<t}\left(1+b_{k}\right)^{-1} \prod_{0<t_{k}<g_{i}(t)}\left(1+b_{k}\right)^{\alpha} p_{i}(t) d t=\infty .
$$

If for any constant $c>0$,

$$
\int_{0}^{\infty} \sum_{i=1}^{m} \prod_{0<i_{k}<t}\left(1+b_{k}\right)^{-1} f_{i}\left(t, c \prod_{0<i_{k}<g_{i}(t)}\left(1+b_{k}\right)\right) d t=\infty
$$

and

$$
\int_{0}^{\infty} \sum_{i=1}^{m} \prod_{0<i_{k}<t}\left(1+b_{k}\right)^{-1} f_{i}\left(t,-c \prod_{0<i_{k}<g_{i}(t)}\left(1+b_{k}\right)\right) d t=-\infty
$$

then all proper solutions of (1.1) with (1.2) are oscillatory.

PROOF. From Theorem 2.2 and the remark, we only need to prove that all proper solutions of $(*)$ are oscillatory. Let $x(t)$ be a nonoscillatory solution of $(*)$. Without loss of generality we may suppose that $x(t)$ is eventually positive. Thus there exists $T>0$ such that $x(t)>0, x\left(g_{i}(t)\right)>0, i=1,2, \ldots, m$, for all $t \geq T$. So 
$\lim _{t \rightarrow \infty} x(t)=L \geq 0$. Suppose that $L>0$. Then there exists $T_{1} \geq T$ such that for all $t \geq T_{1}, x\left(g_{i}(t)\right)>L / 2, i=1,2, \ldots, m$. Hence it follows from $(*)$ that

$$
x^{\prime}(t)+\sum_{i=1}^{m} \prod_{\sigma<t_{k}<t}\left(1+b_{k}\right) f_{i}\left(t, \frac{L}{2} \prod_{\sigma<t_{k}<l}\left(1+b_{k}\right)\right) \leq 0 \text { a.e. }
$$

Integrating the above inequality from $T_{1}$ to $t$, then letting $t \rightarrow \infty$ we have

$$
L-x\left(T_{1}\right)+\int_{T_{1}}^{\infty} \sum_{i=1}^{m} \prod_{\sigma<t_{k}<t}\left(1+b_{k}\right)^{-1} f\left(t, \frac{L}{2} \prod_{\sigma<i_{k}<g_{i}(t)}\left(1+b_{k}\right)\right) d t \leq 0,
$$

that is,

$$
L-x\left(T_{1}\right)+B \int_{T_{1}}^{\infty} \sum_{i=1}^{m} \prod_{0<t_{k}<t}\left(1+b_{k}\right)^{-1} f_{i}\left(t, L_{1} \prod_{0<t_{k}<s_{i}(t)}\left(1+b_{k}\right)\right) d t \leq 0,
$$

where $B=\prod_{0<i_{k} \leq \sigma}\left(1+b_{k}\right)$ and $L_{1}=(L / 2) \prod_{0<<_{k} \leq \sigma}\left(1+b_{k}\right)^{-1}$. Equation (2.9) contradicts (2.7). Hence $L=0$. Therefore it follows from $(*)$ and $\left(\mathrm{A}_{4}\right)$ that there exists $T_{2} \geq T_{1}$ such that for $t \geq T_{2}, 0<x\left(g_{i}(t)\right) \leq \delta, i=1,2, \ldots, m$, and

$$
\frac{x^{\prime}(t)}{x^{\alpha}(t)}+\sum_{i=1}^{m} \prod_{\sigma<t_{k}<t}\left(1+b_{k}\right)^{-1} \prod_{\sigma<t_{k}<g_{i}(t)}\left(1+b_{k}\right)^{\alpha} p_{i}(t) \leq 0 \text { a.e. } t \geq T_{2} .
$$

Integrating the above inequality from $T_{2}$ to $t$ and letting $t \rightarrow \infty$ we find

$$
-(1-\alpha) x\left(T_{2}\right)^{-\alpha+1}+\int_{T_{2}}^{\infty} \sum_{i=1}^{m} \prod_{\sigma<t_{k}<t}\left(1+b_{k}\right)^{-1} \prod_{\sigma<t_{k}<\delta_{i}(t)}\left(1+b_{k}\right)^{\alpha} p_{i}(t) d t \leq 0 .
$$

Clearly, the above inequality contradicts (2.6). The proof of Theorem 2.5 is thus complete.

By combining Theorems 2.3 and 2.5 one easily obtains the following result.

THEOREM 2.6. Assume that $\left(\mathrm{A}_{1}\right)-\left(\mathrm{A}_{4}\right)$ hold. Then all proper solutions of $(1.1)$ with (1.2) are oscillatory if and only if $(2.6)-(2.8)$ are satisfied.

Let

$$
f_{i}(t, u)= \begin{cases}p_{i}(t) u|u|^{\alpha-1}, & u \neq 0 \text { and } 0<\alpha<1, \\ 0, & u=0,\end{cases}
$$

where $p_{i}(t) \in([0, \infty),[0, \infty)), i=1,2, \ldots, m$, are Lebesgue measurable and essentially bounded in each finite interval.

From Theorem 2.6, we obtain the following result.

COROLLARY 2.7. Assume that $\left(\mathrm{A}_{1}\right)-\left(\mathrm{A}_{3}\right)$ and $(2.10)$ hold. Then all proper solutions of (1.1) with (1.2) are oscillatory if and only if (2.6) is satisfied. 


\section{Applications}

In this section we consider the following equation:

$$
\begin{aligned}
& N^{\prime}(t)=r(t) N(t)\left(1-\frac{N(g(t))}{K}\right)\left|1-\frac{N(g(t))}{K}\right|^{\alpha-1}, \quad t \neq t_{k} \text { a.e. } t \geq 0, \\
& N\left(t_{k}^{+}\right)=N\left(t_{k}\right)\left(\frac{N\left(t_{k}\right)}{K}\right)^{b_{k}}, \quad k \in \mathcal{N}, b_{k} \in(-1, \infty),
\end{aligned}
$$

where

$\left(\mathrm{A}_{5}\right) \quad r(t) \in([0, \infty),(0, \infty))$ is Lebesgue measurable and essentially bounded in each finite interval, $K$ and $\alpha$ are positive constants with $0<\alpha<1$.

(A $\left.\mathrm{A}_{6}\right) g(t) \in([0, \infty),[0, \infty))$ is Lebesgue measurable and $g(t)<t, \lim _{t \rightarrow \infty} g(t)=\infty$.

When $b_{k}=0, k \in \mathscr{N}$ in (3.2), (3.1) with (3.2) reduces to a delay differential equation without impulse effects:

$$
N^{\prime}(t)=r(t) N(t)\left(1-\frac{N(g(t))}{K}\right)\left|1-\frac{N(g(t))}{K}\right|^{\alpha-1}, \quad t \geq 0,
$$

which has been of some interest in mathematical ecology for the simulation of population dynamics, where $N$ is the population number and $K$ is the capacity of the environment. This has been studied in [1], [7] and [14].

DEFINITION 4. A proper solution $N(t)$ of (3.1) with (3.2) is said to be oscillatory about $K$ if $N(t)-K$ is neither eventually positive nor eventually negative. Otherwise, it is called nonoscillatory about $K$.

THEOREM 3.1. Assume that $\left(\mathrm{A}_{3}\right),\left(\mathrm{A}_{5}\right)$ and $\left(\mathrm{A}_{6}\right)$ hold. Then all positive solutions of (3.1) with (3.2) are oscillatory about $K$ if and only if

$$
\int_{0}^{\infty} \prod_{0<t_{k}<t}\left(1+b_{k}\right)^{-1} \prod_{0<t_{k}<g(t)}\left(1+b_{k}\right)^{\alpha} r(t) d t=\infty .
$$

ProOF. Let $N(t)=K e^{y(t)}$. From (3.1) and (3.2) we obtain

$$
\begin{aligned}
& y^{\prime}(t)+r(t)\left(e^{y(g(t))}-1\right)\left|e^{y(g(t))}-1\right|^{\alpha-1}=0, \quad t \neq t_{k} \text { a.e. } t \geq 0, \\
& y\left(t_{k}^{+}\right)-y\left(t_{k}\right)=b_{k} y\left(t_{k}\right), \quad k \in \mathscr{N} .
\end{aligned}
$$

Clearly, the solution of (3.1) with (3.2) is oscillatory about $K$ if and only if the solution of (3.4) with (3.5) is oscillatory. Since there is a constant $\delta>0$ such that for all sufficiently large $t$ and $0<|u|<\delta$

$$
\inf _{0<|u|<\delta} \frac{r(t)\left(e^{u}-1\right)\left|e^{u}-1\right|^{\alpha-1}}{u|u|^{\alpha-1}} \geq r(t),
$$


it follows from Theorem 2.6 that we can conclude that all proper solutions of (3.4) with (3.5) are oscillatory if and only if (3.3) holds. The proof of Theorem 3.1 is complete.

Applying Theorem 2.6 to the nonimpulsive equation ( $\star$ ), one can obtain the following result.

COROLLARY 3.2. Assume that $\left(\mathrm{A}_{5}\right)$ and $\left(\mathrm{A}_{6}\right)$ hold. Then all positive solutions of $(\star)$ are oscillatory about $K$ if and only if $\int_{0}^{\infty} r(t) d t=\infty$.

REMARK. Corollary 3.2 extends [7, Theorem 2.8.6], but uses different techniques.

EXAMPLE 1. Consider (3.1) with (3.2). Let $t_{k}=k, b_{k}=-k /(k+1), k \in \mathscr{N}$, $g(t)=t-1, r(t)=(t+1)^{-\beta}, 1<\beta<2,0<\alpha<1$. Thus we have

$$
\int_{0}^{t} \prod_{0<t_{k}<s}\left(1+b_{k}\right)^{-1} \prod_{0<t_{k}<s-1} \frac{\left(1+b_{k}\right)^{\alpha}}{(s+1)^{\beta}} d s \geq \int_{0}^{|t|} \prod_{0<t_{k}<s-1} \frac{(k+1)^{1-\alpha}}{(s+1)^{\beta}} d s,
$$

where $[t]$ denotes the greatest integer function. Hence

$$
\begin{aligned}
\int_{0}^{[t]} \prod_{0<t_{k}<s-1} \frac{(k+1)^{1-\alpha}}{(s+1)^{\beta}} d s & \geq \sum_{k=1}^{[t]-1} \int_{k-1}^{k} \frac{[(k+1) !]^{1-\alpha}}{(s+1)^{\beta}} d s \\
& \geq \sum_{k=1}^{[t]-1} \frac{[(k+1) !]^{1-\alpha}}{(k+1)^{\beta}} \rightarrow \infty \text { as } t \rightarrow \infty .
\end{aligned}
$$

Therefore, by Theorem 3.1, all proper solutions of (3.1) with (3.2) are oscillatory about $K$.

However, when $b_{k}=0, k \in \mathscr{N}$, (3.1) with (3.2) reduces to the following delay differential equation without impulse effects:

$$
N^{\prime}(t)=\frac{1}{(t+1)^{\beta}} N(t)\left(1-\frac{N(t-1)}{K}\right)\left|1-\frac{N(t-1)}{K}\right|^{\alpha-1}, \quad t \geq 0 .
$$

Since $\int_{0}^{\infty}(t+1)^{-\beta} d t<\infty$, it readily follows from Corollary 3.2 that (3.6) has at least one nonoscillatory solution about $K$. This fact demonstrates that the oscillation of impulsive delay differential equations can be caused by impulse effects.

\section{Acknowledgements}

This work was supported by the NNSF of China, the NSF of Shanxi Province and the Tianyuan Foundation of China (10226004). The authors thank the referee for useful comments and suggestions. 


\section{References}

[1] W. G. Aiello, "The existence of nonoscillatory solutions to a generalized, nonautonomous delay logistic equation", J. Math. Anal. Appl. 149 (1990) 114-123.

[2] D. D. Bainov and I. M. Stamova, "Stability of the solutions of impulsive functional-differential equations by Lyapunov's direct method", ANZIAM J. 43 (2001) 269-278.

[3] D. D. Bainov and I. M. Stamova, "Vector Lyapunov functions and conditional stability for systems of impulsive differential-difference equations", ANZIAM J. 42 (2001) 341-353.

[4] G. Ballinger and Xinzhi Liu, "Existence, uniqueness and boundedness results for impulsive delay differential equations", Appl. Anal. 74 (2000) 71-93.

[5] L. Berezansky and E. Braverman, "Oscillation of a linear delay impulsive differential equation", Comm. Appl. Nonlinear Anal. 3 (1996) 61-77.

[6] Y. Duan, W. Feng and J. Yan, "Linearized oscillation of nonlinear impulsive delay differential equations", Computers Math. Applic. 44 (2002) 1267-1274.

[7] L. H. Erbe, Q. K. Kong and B. G. Zhang, Oscillation theory for functional differential equations (Marcel Dekker, New York, 1995).

[8] K. Gopalsamy, Stability and oscillation in delay differential equations of population dynamics (Kluwer, Dordrecht, 1992).

[9] K. Gopalsamy and B. G. Zhang, "On delay differential equation with impulses", J. Math. Anal. Appl. 139 (1989) 110-122.

[10] I. Györi and G. Ladas, Oscillation theory of delay differential equations with applications (Clarendon, Oxford, 1991).

[11] J. Hale, Theory of functional differential equations (Springer, New York, 1977).

[12] V. Lakshmikantham, D. D. Bainov and P. S. Simeonov, Theory of impulsive differential equations (World Scientific, Singapore, 1989).

[13] J. Shen, "Global existence and uniqueness, oscillation and nonoscillation of impulsive delay differential equations", Acta Math. Sinica 40 (1997) 53-59.

[14] Z. C. Wang, J. S. Yu and L. H. Huang, "Nonoscillatory solutions of generalized delay logistic equations", Chinese J. Math. 21 (1993) 81-90.

[15] J. Yan and A. Zhao, "Oscillation and stability of linear impulsive delay differential equations", $J$. Math. Anal. Appl. 227 (1998) 187-194. 